\title{
Pullback D-Attractor of Coupled Rod Equations with Nonlinear Moving Heat Source
}

\author{
Danxia Wang, Jianwen Zhang, and Yinzhu Wang \\ Department of Mathematics, Taiyuan University of Technology, Taiyuan 030024, China \\ Correspondence should be addressed to Jianwen Zhang; jianwen.z2008@163.com
}

Received 12 February 2014; Accepted 9 May 2014; Published 3 July 2014

Academic Editor: Bo-Qing Dong

Copyright ( 2014 Danxia Wang et al. This is an open access article distributed under the Creative Commons Attribution License, which permits unrestricted use, distribution, and reproduction in any medium, provided the original work is properly cited.

\begin{abstract}
We consider the pullback $D$-attractor for the nonautonomous nonlinear equations of thermoelastic coupled rod with a nonlinear moving heat source. By Galerkin method, the existence and uniqueness of global solutions are proved under homogeneous boundary conditions and initial conditions. By prior estimates combined with some inequality skills, the existence of the pullback $D$-absorbing set is obtained. By proving the properties of compactness about the nonlinear operator $g_{1}(\cdot), g_{2}(\cdot)$, and then proving the pullback $D$-condition $(\mathrm{C})$, the existence of the pullback $D$-attractor of the equations previously mentioned is given.
\end{abstract}

\section{Introduction}

In this paper, we consider a thermoelastic coupled rod system:

$$
\begin{gathered}
u_{t t}-\beta \Delta u+\gamma u_{t}+\nabla \widetilde{\theta}+g_{1}(u)=f(x, t), \\
\tilde{\theta}_{t}-k \Delta \widetilde{\theta}+\nabla u_{t}=g_{2}(\widetilde{\theta})+Q(x, t), \\
\left.u\right|_{\partial \Omega}=0,\left.\quad \nabla \widetilde{\theta}\right|_{\partial \Omega}=0, \quad x \in \partial \Omega \\
u(x, \tau)=u_{0}(x), \quad u_{t}(x, \tau)=p_{0}(x), \quad \widetilde{\theta}(x, \tau)=\widetilde{\theta}_{0}(x), \\
x \in \Omega
\end{gathered}
$$

with an external force function and a nonlinear moving heat source function. Here $u(x, t)$ is the rod elastic displacement. $\widetilde{\theta}(x, t)$ is the dimensionless temperature. $\beta, \gamma, k$ are all positive constants, where $\beta$ is the square of wave velocity, $\gamma$ is the damping coefficient, and $k$ is the thermal diffusivity. $\Omega \subset R^{2}$ is a bounded smooth domain. $f(x, t)$ is the external force and $f(x, t)$ is locally square integrable with respect to time for $t \in R, x \in \Omega$; that is, $f(x, t) \in L_{\text {loc }}^{2}\left(R, L^{2}(\Omega)\right) . Q(x, t)$ is the moving heat source and $Q(x, t)$ is locally square integrable in time for $t \in R, x \in \Omega$; that is, $Q(x, t) \in L_{\text {loc }}^{2}\left(R, L^{2}(\Omega)\right)$. $g_{1}(u)$ and $g_{2}(\tilde{\theta})$ are all the nonlinear function, and $g_{1}(u)$ and $g_{2}(\tilde{\theta})$ are continuous on $R$, respectively. We give the pullback $D$-attractor for the nonautonomous nonlinear equations of thermoelastic coupled rod in space $E_{1}=D(A) \times V \times V$, where $A=-\Delta, V=H_{0}^{1}(\Omega)$.

Recently the research of the nonautonomous infinite dimensional dynamical system has been paid much attention and developed fast as evidence by the references cited in [17]. Chepyzhov and Vishik [1] firstly extend the notion of global attractor in the autonomous case to the concept of the uniform attractor for the nonautonomous case. But the uniform attractor is not applicable to the nonautonomous systems in which the trajectories can be unbounded as time increases to infinity. Therefore some new concepts and theories must be brought up for such nonautonomous case, where the concepts and the theorem of existence of the pullback $D$-attractor were advanced in [2-8] and so on.

Caraballo et al. [2] and so forth gave the existence of the pullback $D$-attractor for a nonautonomous N-S equation under the assumptions of asymptotic compactness and existence of a family of absorbing sets. Wang and Zhong [3] advanced the existence of the pullback $D$-attractor for the dissipative Sine-Gordon wave equation in an unbounded domain in which the external force did not need to be bounded. In $[4,5]$, The author studied the pullback attractor of the reaction-diffusion equation and the generalized Korteweg-de Vries-Burgers equation, respectively. S. H. Park 
and J. Y. Park [6] considered the nonautonomous modified Swift-Hohenberg equation

$$
u_{t}+\Delta^{2} u+2 \Delta u+a u+b|\nabla u|^{2}+u^{3}=g(x, t)
$$

and proved the existence of the pullback attractor when its external force has exponential growth. The abovementioned systems are all specific systems. For the widespread used nonautonomous structural system in engineering, the study has been paid less attention. Park and Kang [7] studied the existence of the pullback $D$-attractor for nonautonomous suspension bridge equation because of being motivated by Ma et al. $[8,9]$ :

$$
u_{t t}+\Delta^{2} u+\mu u_{t}+k u^{+}+g(u)=f(x, t) .
$$

In this paper, based on Al-Huniti et al. [10] as the relaxation time $\bar{\tau}$ is not considered and Carlson [11], we study a more general nonlinear thermoelastic coupled system (1)-(4) of a rod due to a nonlinear moving heat source $Q(x, t)$. We give the existence of a pullback $D$-attractor of above system by proving the existence of a pullback $D$-absorbing set and pullback condition (C) for the external force $f(x, t)$ unnecessarily bounded.

In fact, we assume that the external forces $f(x, t)$ and $Q(x, t)$ satisfy $f(x, t) \in L_{\text {loc }}^{2}\left(R, L^{2}(\Omega)\right), f^{\prime}(x, t) \quad \epsilon$ $L_{\text {loc }}^{2}\left(R, L^{2}(\Omega)\right)$, and $Q(x, t) \in L_{\text {loc }}^{2}\left(R, L^{2}(\Omega)\right)$, respectively, and for any $t \in R$

$$
\int_{-\infty}^{t} e^{\delta s}\left(|f(s)|^{2}+\left|f^{\prime}(s)\right|^{2}+|Q(s)|^{2}\right) d s<\infty,
$$

where $\delta>0$ is a small real number which will be characterized later.

On the assumptions of the nonlinear function $g_{1}(\cdot)$, Park and Kang gave the assumption

$$
\underset{|s| \rightarrow \infty}{\lim \sup _{\mid}} \frac{\left|g_{1}^{\prime}(s)\right|}{|s|^{\gamma}}=0,
$$

(where $0 \leq \gamma<\infty$ ) for nonautonomous suspension bridge equations in [7]. At present, we remove the assumption of [7] and we assume that the nonlinear function $g_{1}(\cdot) \in C^{2}(R, R)$ satisfies the following assumptions:

$\left(H_{1}\right)$ we denote by $G(s)$ the primitive of $g_{1}(s)$; that is, $G(s)=$ $\int_{0}^{s} g_{1}(\tau) d \tau$, and then

$$
\liminf _{|s| \rightarrow \infty} \frac{|G(s)|}{s^{2}} \geq 0
$$

$\left(H_{2}\right) g_{1}(u) \leq 1+|u|^{\rho+1}$ for some $0 \leq \rho<\infty$;

$\left(H_{3}\right) g_{1}^{\prime}(u) \leq C^{\prime}\left(1+|u|^{\rho}\right)$ for some $0 \leq \rho<\infty$;

$\left(H_{4}\right)$ there exists a constant $C_{0}>0$ such that

$$
\liminf _{|s| \rightarrow \infty} \frac{s g_{1}(s)-C_{0} G(s)}{s^{2}} \geq 0
$$

$\left(H_{5}\right) g_{1}(0)=0$
We also assume that the nonlinear function $g_{2}(\cdot) \epsilon$ $C^{1}(R, R)$ satisfies the following assumptions: there exists a constant $a_{1}$ such that

$$
g_{2}(0)=0, \quad\left|g_{2}^{\prime}(s)\right| \leq a_{1}, \quad \forall s \in R .
$$

Throughout this paper, we introduce the spaces $H=$ $L^{2}(\Omega)$ and $V=H_{0}^{1}(\Omega)$ and endow these spaces with the usual scalar products and norms $(\cdot, \cdot),|\cdot|,((\cdot, \cdot)),\|\cdot\|$, where $(u, v)=\int_{\Omega} u v d x,((u, v))=\int_{\Omega} \nabla u \nabla v d x$. Because of defining $A=-\Delta$, with reference to [7] we have the scalar products $(A u, A u)$ and norm $|A u|^{2}$ in the space $D(A)$. By the Poincare inequality, there exists a proper constant $\lambda_{1}, \lambda_{2}>0$ such that

$$
\begin{aligned}
& \|u\|^{2} \geq \lambda_{1}|u|^{2}, \quad \forall u \in V \\
& |A u|^{2} \geq \lambda_{2}\|u\|^{2}, \quad \forall u \in D(A) .
\end{aligned}
$$

\section{Pullback $D_{\delta, E_{1}}$-Attracting Set}

By normal Galerkin method (see [1, 12-14]), we have the following theorem of existence and uniqueness of solutions to problems (1)-(4).

Theorem 1. Assume that $\beta, \gamma, k>0, f(x, t), Q(x, t) \in$ $L_{\text {loc }}^{2}(R, H)$ and the assumptions of the functions $g_{1}(\cdot), g_{2}(\cdot)$ hold; then, for all $T>0$ and any given $u_{0} \in V, p_{0} \in H, \widetilde{\theta}_{0} \in H$, problems (1)-(4) have a unique solution $(u, \widetilde{\theta})$ such that

$$
u \in C^{0}\left(R_{\tau} ; V\right) \cap C^{1}\left(R_{\tau} ; H\right), \quad \tilde{\theta} \in C^{0}\left(R_{\tau} ; H\right),
$$

where $R_{\tau}=[\tau, \infty\}$

Moreover $f^{\prime}(x, t) \in L_{l o c}^{2}(\tau, T ; H)$, for all $T>0, u_{0} \in D(A)$, $p_{0} \in V, \widetilde{\theta}_{0} \in V$; then

$$
u \in C^{0}\left(R_{\tau} ; D(A)\right) \cap C^{1}\left(R_{\tau} ; V\right), \quad \widetilde{\theta} \in C^{0}\left(R_{\tau} ; V\right) .
$$

For simplicity, we write $y(r)=\left(u(r), \partial_{r} u(r), \widetilde{\theta}(r)\right)=$ $(u(r), p(r), \tilde{\theta}(r)), y_{0}=\left(u_{0}, p_{0}, \widetilde{\theta}_{0}\right)$. We denote by $E_{0}=V \times$ $H \times H$ the space of vector functions $y(r)=(u(r), p(r), \widetilde{\theta}(r))$ with the norm $\|y\|_{E_{0}}=\|u\|^{2}+|p|^{2}+|\widetilde{\theta}|^{2}$ in $E_{0}$ and denote by $E_{1}=D(A) \times V \times V$ the space of vector functions $y(r)=$ $(u(r), p(r), \widetilde{\theta}(r))$ with the norm $\|y\|_{E_{1}}=|A u|^{2}+\|p\|^{2}+\|\widetilde{\theta}\|^{2}$ in $E_{1}$. We can construct the nonautonomous dynamical system generated by problems (1)-(4) in $E_{0}$ or $E_{1}$. We consider $Q=$ $R, \theta_{t} \tau=\tau+t$, and then we define

$$
\begin{aligned}
\Phi\left(t, \tau, y_{0}\right)= & y\left(t+\tau, \tau, y_{0}\right) \\
= & (u(t+\tau), p(t+\tau), \widetilde{\theta}(t+\tau)), \\
& \quad \tau \in R, \quad t \geq 0, \quad y_{0} \in E_{0}, E_{1} .
\end{aligned}
$$

The uniqueness of solutions to problems (1)-(4) implies that

$$
\begin{array}{r}
\Phi\left(t, \tau, y_{0}\right)=\Phi\left(t, s+\tau, \Phi\left(s, \tau, y_{0}\right)\right) \\
\tau \in R, \quad t \geq 0, \quad y_{0} \in E_{0}, E_{1} .
\end{array}
$$


And, for all $\tau \in R, t \geq 0$, the mapping $\Phi(t, \tau, \cdot): E_{0} \rightarrow E_{0}$ (or $E_{1} \rightarrow E_{1}$ ) defined by (15) is continuous. Consequently, the mapping $\Phi(t, \tau, \cdot)$ defined by (15) is a continuous cocycle on $E_{0}$ or $E_{1}$. that

Let $\mathfrak{R}_{\delta}$ be the set of all functions $r: R \rightarrow(0,+\infty)$ such

$$
\lim _{t \rightarrow \infty} e^{\delta t} r^{2}(t)=0
$$

where $0<\delta<2 \alpha_{1}$ and $\alpha_{1}=\min \left\{3 \alpha / 16, \gamma / 2, k \lambda / 4, \alpha C_{0} / 2\right\}$ and $D_{\delta, E_{0}}$ denotes the class of all families $\widehat{D}=\{D(t) ; t \in R\} \subset$ $P\left(E_{0}\right)$ such that $D(t) \subset \bar{B}\left(0, r_{\widehat{D}}(t)\right)$ for some $r_{\widehat{D}} \in \Re_{\delta}$, where $\bar{B}\left(0, r_{\widehat{D}}(t)\right)$ denotes the closed ball in $E_{0}$ centered on 0 with radius $r_{\widehat{D}}(t)$.

Theorem 2. Assume that $\beta, \gamma, k>0$ and the assumptions of the functions $g_{1}(\cdot), g_{2}(\cdot)$ hold. Suppose that $f(x, t) \in$ $L_{l o c}^{2}(R, H)$ and $Q(x, t) \in L_{l o c}^{2}(R, H)$ satisfy (7). Then there exists a pullback $D_{\delta, E_{0}}$-attracting set in $E_{0}$ for the nonautonomous dynamical system $(\theta, \Phi)$ defined by (15).

Proof. Let $t \in R, \tau \geq 0$, and $y_{0}=\left(u_{0}, p_{0}, \widetilde{\theta}_{0}\right) \in E_{0}$ be fixed. Define

$$
\begin{gathered}
u(r)=u\left(r, t-\tau, u_{0}\right), \\
p(r)=u^{\prime}\left(r, t-\tau, p_{0}\right), \\
\tilde{\theta}(r)=\tilde{\theta}\left(r, t-\tau, \tilde{\theta}_{0}\right), \\
\quad \text { for } r \geq t-\tau, \\
(u(r), p(r), \tilde{\theta}(r))=\Phi\left(r-t+\tau, t-\tau, y_{0}\right) \\
\quad \text { for } r \geq t-\tau .
\end{gathered}
$$

Taking the scalar product in $H$ of (1) with $v=u^{\prime}+\alpha u$ and taking the scalar product in $H$ of (2) with $\tilde{\theta}$, after a computation of addition, we obtain

$$
\begin{aligned}
\frac{1}{2} \frac{d}{d r} & \left(\beta\|u\|^{2}+|v|^{2}+|\widetilde{\theta}|^{2}\right)+\gamma|v|^{2}-\alpha|v|^{2} \\
& +\alpha \beta\|u\|^{2}+k\|\widetilde{\theta}\|^{2}+\alpha^{2}(u, v) \\
& -\gamma \alpha(u, v)+\alpha(\nabla \widetilde{\theta}, u)+\left(g_{1}(u), v\right) \\
= & (f, v)+\left(g_{2}(\widetilde{\theta}), \widetilde{\theta}\right)+(Q, \widetilde{\theta}) .
\end{aligned}
$$

For simplicity, define $\phi(u)=\int_{\Omega} G(u) d \Omega$. By the assumption $\left(H_{1}\right)$, it is obvious that $\phi(u) \geq 0$. By the assumption $\left(H_{4}\right)$ of $g_{1}(\cdot)$, we have

$$
\left(g_{1}(u), u\right)-C_{0} \int_{\Omega} G(u) d \Omega+\frac{\lambda}{16}|u|^{2} \geq-M
$$

so

$$
\begin{aligned}
& \left(g_{1}(u), v\right) \\
& \quad=\left(g_{1}(u), u^{\prime}\right)+\alpha\left(g_{1}(u), u\right) \\
& \quad \geq \int_{\Omega} \frac{d}{d r} G(u) d \Omega+\alpha C_{0} \int_{\Omega} G(u) d \Omega-\alpha \frac{\lambda}{16}|u|^{2}-\alpha M \\
& \quad \geq \frac{d}{d r} \phi(u)+\alpha C_{0} \phi(u)-\alpha \frac{\lambda}{16}|u|^{2}-\alpha M .
\end{aligned}
$$

Considering assumption (11) of $g_{2}(\cdot)$, we have

$$
\left|\left(g_{2}(\widetilde{\theta}), \widetilde{\theta}\right)\right| \leq\left|g_{2}(\widetilde{\theta})\right||\widetilde{\theta}| \leq a_{1}|\widetilde{\theta}|^{2} .
$$

By the Young inequality and (12), we have

$$
\begin{aligned}
& |(f, v)| \leq \frac{1}{\gamma}|f|^{2}+\frac{\gamma}{4}|v|^{2} ; \\
& |(Q, \widetilde{\theta})| \leq \frac{1}{\lambda k}|Q|^{2}+\frac{k \lambda}{4}|\widetilde{\theta}|^{2} \leq \frac{1}{\lambda k}|Q|^{2}+\frac{k}{4}\|\widetilde{\theta}\|^{2}, \\
& \alpha^{2}(u, v) \geq-\frac{\alpha^{2}}{2}|u|^{2}-\frac{\alpha^{2}}{2}|v|^{2} ; \\
& -\gamma \alpha(u, v) \geq-\frac{2 \gamma^{2} \alpha}{\lambda}|v|^{2}-\frac{\alpha \lambda}{8}|u|^{2} ; \\
& \alpha(\nabla \widetilde{\theta}, u) \geq-\frac{\alpha}{2 \beta}|\widetilde{\theta}|^{2}-\frac{\alpha \beta}{2}\|u\|^{2} .
\end{aligned}
$$

Letting $0<\alpha \leq \min \left\{\beta \lambda / 2+\lambda / 4,-\left(1+2 \gamma^{2} / \lambda\right)+\right.$ $\left.\sqrt{\left(2 \gamma^{2} / \lambda+1\right)^{2}+\gamma / 2}, k \lambda \beta / 2,1\right\}$ and taking $a_{1} / k<\lambda / 4$ and $\beta \geq 1$, we infer from (19) that

$$
\begin{aligned}
\frac{1}{2} \frac{d}{d r} & \left(\beta\|u\|^{2}+|v|^{2}+|\widetilde{\theta}|^{2}+2 \phi(u)\right) \\
& +\frac{3 \alpha \beta}{16}\|u\|^{2}+\frac{\gamma}{2}|v|^{2}+\frac{k \lambda}{4}|\widetilde{\theta}|^{2}+\alpha C_{0} \phi(u) \\
\leq & \frac{1}{\gamma}|f|^{2}+\alpha M+\frac{1}{\lambda k}|Q|^{2} .
\end{aligned}
$$

Also taking $\alpha_{1}=\min \left\{3 \alpha / 16, \gamma / 2, k \lambda / 4, \alpha C_{0} / 2\right\}$, we have

$$
\begin{aligned}
& \frac{d}{d r}\left(\beta\|u\|^{2}+|v|^{2}+|\widetilde{\theta}|^{2}+2 \phi(u)\right) \\
& +2 \alpha_{1}\left(\beta\|u\|^{2}+|v|^{2}+|\widetilde{\theta}|^{2}+2 \phi(u)\right) \\
& \leq \frac{2}{\gamma}|f|^{2}+2 \alpha M+\frac{2}{\lambda k}|Q|^{2}
\end{aligned}
$$

Note that

$$
\begin{aligned}
& \frac{d}{d r} e^{\delta r}\left(\beta\|u\|^{2}+|v|^{2}+|\widetilde{\theta}|^{2}+2 \phi(u)\right) \\
&= \delta e^{\delta r}\left(\beta\|u\|^{2}+|v|^{2}+|\widetilde{\theta}|^{2}+2 \phi(u)\right) \\
&+e^{\delta r} \frac{d}{d r}\left(\beta\|u\|^{2}+|v|^{2}+|\widetilde{\theta}|^{2}+2 \phi(u)\right),
\end{aligned}
$$


and by (25), we have

$$
\begin{aligned}
\frac{d}{d r} e^{\delta r}\left(\beta\|u\|^{2}+|v|^{2}+|\widetilde{\theta}|^{2}+2 \phi(u)\right) \\
\leq\left(\delta-2 \alpha_{1}\right) e^{\delta r}\left(\beta\|u\|^{2}+|v|^{2}+|\widetilde{\theta}|^{2}+2 \phi(u)\right) \\
+e^{\delta r}\left(\frac{2}{\gamma}|f|^{2}+2 \alpha M+\frac{2}{\lambda k}|Q|^{2}\right) .
\end{aligned}
$$

By integrating (27) over the interval $[t-\tau, t]$, we obtain

$$
\begin{gathered}
e^{\delta t}\left(\beta\|u(t)\|^{2}+|v(t)|^{2}+|\widetilde{\theta}(t)|^{2}+2 \phi(u)\right) \\
\leq e^{\delta(t-\tau)}\left(\beta\|u(t-\tau)\|^{2}+|v(t-\tau)|^{2}\right. \\
\left.+|\widetilde{\theta}(t-\tau)|^{2}+2 \phi(u(t-\tau))\right) \\
+\int_{t-\tau}^{t} e^{\delta s}\left(\frac{2}{\gamma}|f|^{2}+\frac{2}{\lambda k}|Q|^{2}\right) d s+\frac{2 \alpha M}{\delta}\left(e^{\delta t}-e^{\delta(t-\tau)}\right) \\
+\int_{t-\tau}^{t}\left(\delta-2 \alpha_{1}\right) e^{\delta s}\left(\beta\|u(s)\|^{2}+|v(s)|^{2}\right. \\
\left.+|\widetilde{\theta}(s)|^{2}+2 \phi(u(s))\right) d s .
\end{gathered}
$$

Since $\delta<2 \alpha_{1}$, we have

$$
\begin{aligned}
& \beta\|u(t)\|^{2}+|v(t)|^{2}+|\widetilde{\theta}(t)|^{2}+2 \phi(u(t)) \\
& \leq e^{-\delta \tau}\left(\beta\|u(t-\tau)\|^{2}+|v(t-\tau)|^{2}\right. \\
& \left.\quad+|\widetilde{\theta}(t-\tau)|^{2}+2 \phi(u(t-\tau))\right) \\
& \quad+e^{-\delta t} \int_{t-\tau}^{t} e^{\delta s}\left(\frac{2}{\gamma}|f|^{2}+\frac{2}{\lambda k}|Q|^{2}\right) d s+\frac{2 \alpha M}{\delta}\left(1-e^{-\delta \tau}\right) .
\end{aligned}
$$

Note that

$$
\begin{aligned}
& \|u\|^{2}+|p|^{2}+|\widetilde{\theta}|^{2}+2 \phi(u) \\
& \quad \leq\left(1+\frac{2 \alpha^{2}}{\lambda \beta}\right)\left(\beta\|u\|^{2}\right)+2|v|^{2}+|\widetilde{\theta}|^{2}+2 \phi(u) .
\end{aligned}
$$

If we take $C_{1}=\max \left\{2,1+2 \alpha^{2} / \lambda \beta\right\}$, we infer from (29) that

$$
\begin{aligned}
&\|u\|^{2}+|p|^{2}+|\widetilde{\theta}|^{2}+2 \phi(u) \\
& \leq C_{1}\left(\beta\|u\|^{2}+|v|^{2}+|\widetilde{\theta}|^{2}+2 \phi(u)\right) \\
& \leq C_{1} e^{-\delta \tau}\left(\beta\|u(t-\tau)\|^{2}+|v(t-\tau)|^{2}\right. \\
&\left.+|\widetilde{\theta}(t-\tau)|^{2}+2 \phi(u(t-\tau))\right)
\end{aligned}
$$

$$
\begin{aligned}
& +C_{1} e^{-\delta t} \int_{t-\tau}^{t} e^{\delta s}\left(\frac{2}{\gamma}|f|^{2}+\frac{2}{\lambda k}|Q|^{2}\right) d s \\
& +\frac{2 C_{1} \alpha M}{\delta}\left(1-e^{-\delta \tau}\right) \\
& \leq C_{1} C_{2} e^{-\delta \tau}\left[\|u(t-\tau)\|^{2}+|p(t-\tau)|^{2}\right. \\
& \left.+|\widetilde{\theta}(t-\tau)|^{2}+2 \phi(u(t-\tau))\right] \\
& +C_{1} e^{-\delta t} \int_{t-\tau}^{t} e^{\delta s}\left(\frac{2}{\gamma}|f|^{2}+\frac{2}{\lambda k}|Q|^{2}\right) d s \\
& +\frac{2 C_{1} \alpha M}{\delta}\left(1-e^{-\delta \tau}\right) .
\end{aligned}
$$

Let $\widehat{D}_{\delta, E_{0}}$ be given. For all $y(t-\tau)=y_{0} \in D(t-\tau), t \in R$ and $\tau \geq 0$, from the assumption $\left(H_{4}\right)$ of $g_{1}(\cdot)$, we know that $\phi(u(t-\tau))$ is bounded. So we easily obtain from (31)

$$
\begin{aligned}
& \left\|\Phi\left(t, t-\tau, y_{0}\right)\right\|_{E_{0}}^{2} \\
& \leq C_{1} C_{2} e^{-\delta \tau}\left[\|u(t-\tau)\|^{2}+|p(t-\tau)|^{2}\right. \\
& \left.\quad+|\widetilde{\theta}(t-\tau)|^{2}+2 \phi(u(t-\tau))\right] \\
& +C_{1} e^{-\delta t} \int_{t-\tau}^{t} e^{\delta s}\left(\frac{2}{\gamma}|f|^{2}+\frac{2}{\lambda k}|Q|^{2}\right) d s \\
& +\frac{2 C_{1} \alpha M}{\delta}\left(1-e^{-\delta \tau}\right)
\end{aligned}
$$

for all $y_{0} \in D(t-\tau), t \in R$, and $\tau \geq 0$. Set

$$
\begin{aligned}
\left(R_{\delta}(t)\right)^{2}= & 2 C_{1} e^{-\delta t} \int_{t-\tau}^{t} e^{\delta s}\left(\frac{2}{\gamma}|f|^{2}+\frac{2}{\lambda k}|Q|^{2}\right) d s \\
& +\frac{4 C_{1} \alpha M}{\delta}\left(1-e^{-\delta \tau}\right),
\end{aligned}
$$

and consider the family $\widehat{B}_{\delta, E_{0}}$ of closed balls in $E_{0}$ defined by $B_{\delta}(t)=\left\{y \in E_{0},\|y\|_{E_{0}} \leq R_{\delta}(t)\right\}$. It is easy to check that $\widehat{B}_{\delta, E_{0}} \in$ $D_{\delta, E_{0}}$ and $\widehat{B}_{\delta}$ is pullback $D_{\delta, E_{0}}$-absorbing for the cocycle $\Phi$ by (15).

In order to prove the pullback $D_{\delta, E_{1}}$-attractor, let $R_{\delta}$ be the set of all functions $r: R \rightarrow(0,+\infty)$ which satisfies (17) and $D_{\delta, E_{1}}$ denotes the class of all families $\widehat{D}=\{D(t) ; t \in R\} \subset$ $P\left(E_{1}\right)$ such that $D(t) \subset \bar{B}\left(0, r_{\widehat{D}}(t)\right)$ for some $r_{\widehat{D}} \in \mathfrak{R}_{\delta}$, where $\bar{B}\left(0, r_{\widehat{D}}(t)\right)$ denotes the closed ball in $E_{1}$ centered on 0 with radius $r_{\widehat{D}}(t)$.

Theorem 3. Assume that $\beta, \gamma, k>0$ and the assumptions of $g_{1}(\cdot), g_{2}(\cdot)$ hold. $f(x, t) \in L_{l o c}^{2}\left(R, L^{2}(\Omega)\right), f^{\prime}(x, t) \in$ $L_{\text {loc }}^{2}\left(R, L^{2}(\Omega)\right)$, and $Q(x, t) \in L_{\text {loc }}^{2}\left(R, L^{2}(\Omega)\right)$ satisfy (7). Then there exists a pullback $D_{\delta, E_{1}}$-attracting set in $E_{1}$ for the nonautonomous dynamical system $(\theta, \Phi)$ defined by (15). 
Proof. Let $t \in R, \tau \geq 0$ and $y_{0}=\left(u_{0}, p_{0}, \widetilde{\theta}_{0}\right) \in E_{1}$ be fixed. Take the scalar product in $H$ of (1) with $A v=A u^{\prime}+\alpha A u$, and take the scalar product in $H$ of (2) with $A \widetilde{\theta}$; then make summation to get

$$
\begin{aligned}
\frac{1}{2} \frac{d}{d r} & \left(\beta|A u|^{2}+\|v\|^{2}+\|\widetilde{\theta}\|^{2}\right)+\gamma\|v\|^{2}-\alpha\|v\|^{2} \\
& +\alpha \beta|A u|^{2}+k|A \widetilde{\theta}|^{2}+\alpha^{2}((u, v)) \\
& -\gamma \alpha(A u, v)+\alpha\left(A^{1 / 2} \widetilde{\theta}, A u\right)+\left(g_{1}(u), A v\right) \\
= & \left(g_{2}(\widetilde{\theta}), A \widetilde{\theta}\right)+(f, A v)+(Q, A \widetilde{\theta}) .
\end{aligned}
$$

Since

$$
\begin{aligned}
& \left(g_{1}(u), A v\right) \\
& =\left(g_{1}(u), A u^{\prime}\right)+\alpha\left(g_{1}(u), A u\right) \\
& =\frac{d}{d t}\left(g_{1}(u), A u\right)-\left(g_{1}^{\prime}(u) u^{\prime}, A u\right)+\alpha\left(g_{1}(u), A u\right) ;
\end{aligned}
$$

$$
=\frac{d}{d t}(f, A u)+\alpha(f, A u)-\left(f^{\prime}, A u\right),
$$

we infer from (34) that

$$
\begin{aligned}
& \frac{1}{2} \frac{d}{d r}\left(\beta|A u|^{2}+\|v\|^{2}+\|\widetilde{\theta}\|^{2}+2\left(g_{1}(u), A u\right)-2(f, A u)\right) \\
& \quad+\gamma\|v\|^{2}-\alpha\|v\|^{2}+\alpha \beta|A u|^{2}+k|A \widetilde{\theta}|^{2}+\alpha^{2}(A u, v) \\
& \quad-\gamma \alpha(A u, v)+\alpha\left(A^{1 / 2} \widetilde{\theta}, A u\right)+\alpha\left(g_{1}(u), A u\right)-\alpha(f, A u) \\
& \quad \leq-\left(f^{\prime}, A u\right)+(Q, A \widetilde{\theta})+\left(g_{2}(\widetilde{\theta}), A \widetilde{\theta}\right)+\left(g_{1}^{\prime}(u) u^{\prime}, A u\right) .
\end{aligned}
$$

Also

$$
\begin{aligned}
& \alpha^{2}(A u, v) \geq-\frac{\alpha \beta}{16}|A u|^{2}-\frac{4 \alpha^{3}}{\beta}|v|^{2} ; \\
& -\gamma \alpha(A u, v) \geq-\frac{\alpha \beta}{16}|A u|^{2}-\frac{4 \gamma^{2} \alpha}{\beta}|v|^{2} ; \\
& \alpha\left(A^{1 / 2}(\widetilde{\theta}), A u\right) \geq-\frac{4 \alpha}{\beta}\left|A^{1 / 2} \widetilde{\theta}\right|^{2}-\frac{\alpha \beta}{16}|A u|^{2} ; \\
& -\left(f^{\prime}, A u\right) \leq \frac{4}{\alpha \beta}\left|f^{\prime}\right|^{2}+\frac{\alpha \beta}{16}|A u|^{2} ; \\
& (Q, A \widetilde{\theta}) \leq \frac{2}{k}|Q|^{2}+\frac{k}{8}|A \widetilde{\theta}|^{2} ; \\
& \left(g_{2}(\widetilde{\theta}), A \widetilde{\theta}\right) \leq \frac{2}{k}\left|g_{2}(\widetilde{\theta})\right|^{2}+\frac{k}{8}|A \widetilde{\theta}|^{2}
\end{aligned}
$$

and consider the assumption $\left(H_{3}\right)$ of $g_{1}(\cdot)$ combined with Sobolev-embed theorem

$$
\begin{aligned}
& \left|\left(g_{1}^{\prime}(u) u^{\prime}, A u\right)\right| \\
& \quad=\int_{\Omega} g_{1}^{\prime}(u) u^{\prime} A u d x \\
& \quad \leq\left|C^{\prime}\right| \int_{\Omega}\left|\left(1+|u|^{\rho}\right) u^{\prime} A u\right| d x \\
& \leq \frac{8 C^{\prime 2}}{\alpha \beta}|p|^{2}+\frac{\alpha \beta}{16}|A u|^{2}+\frac{4 C^{\prime 2}}{\alpha \beta} \int_{\Omega}|u|^{2 \rho}\left(u^{\prime}\right)^{2} d x \\
& \leq \frac{8 C^{\prime 2}}{\alpha \beta}|p|^{2}+\frac{\alpha \beta}{16}|A u|^{2}+\gamma\left(\frac{4 C^{\prime 2}}{\alpha \beta}\right)^{2}\|u\|^{2}+\frac{\gamma}{4}\|p\|^{2} \\
& \leq \frac{8 C^{\prime 2}}{\alpha \beta}|p|^{2}+\frac{\alpha \beta}{16}|A u|^{2}+\gamma\left(\frac{4 C^{\prime 2}}{\alpha \beta}\right)^{2}\|u\|^{2} \\
& \quad+\frac{\gamma}{2}\|v\|^{2}+\frac{\alpha^{2} \gamma}{2}\|u\|^{2},
\end{aligned}
$$

and then we infer from (36) that

$$
\begin{aligned}
\frac{1}{2} \frac{d}{d r} & \left(\beta|A u|^{2}+\|v\|^{2}+\|\widetilde{\theta}\|^{2}+2\left(g_{1}(u), A u\right)-2(f, A u)\right) \\
& +\frac{\gamma}{2}\|v\|^{2}-\alpha\|v\|^{2}+\frac{11 \alpha \beta}{16}|A u|^{2}+\frac{3 k}{4}|A \widetilde{\theta}|^{2} \\
& -\frac{4 \alpha}{\beta}\|\widetilde{\theta}\|^{2}+\alpha\left(g_{1}(u), A u\right)-\alpha(f, A u) \\
\leq & \frac{4}{\alpha \beta}\left|f^{\prime}\right|^{2}+\frac{2}{k}|Q|^{2}+\frac{2}{k}\left|g_{2}(\widetilde{\theta})\right|^{2}+\frac{4 \alpha^{3}+4 \alpha \gamma^{2}}{\beta}|v|^{2} \\
& +\left[\frac{\alpha^{2}}{2}+\left(\frac{4 C^{\prime 2}}{\alpha \beta}\right)^{2}\right] \gamma\|u\|^{2}+\frac{8 C^{\prime 2}}{\alpha \beta}|p|^{2} .
\end{aligned}
$$

Let $0<\alpha \leq \min \{3 k \lambda \beta /(16+2 \beta), 3 \gamma / 4\}$. By the Gronwall lemma we have from (39)

$$
\begin{gathered}
\beta|A u|^{2}+\|v\|^{2}+\|\widetilde{\theta}\|^{2}+2\left(g_{1}(u), A u\right)-2(f, A u) \\
\leq e^{-\alpha \tau}\left(\beta\left|A u_{0}\right|^{2}+\left\|v_{0}\right\|^{2}+\left\|\widetilde{\theta}_{0}\right\|^{2}\right. \\
\left.+2\left(g_{1}\left(u_{0}\right), A u_{0}\right)-2\left(f(t-\tau), A u_{0}\right)\right) \\
+\int_{t-\tau}^{t} e^{-\alpha(t-s)}\left\{\frac{8}{\alpha \beta}\left|f^{\prime}\right|^{2}+\frac{4}{k}|Q|^{2}+\frac{4 \alpha_{1}^{2}}{k}|\widetilde{\theta}|^{2}\right. \\
\left.+\frac{8\left(\alpha^{3}+\gamma \alpha^{2}\right)}{\beta}|v|^{2}\right\} d s \\
+\int_{t-\tau}^{t} e^{-\alpha(t-s)}\left\{\left[\left(\frac{\alpha^{2}}{2}+\frac{4 C^{\prime 2}}{\alpha \beta}\right)^{2}\right] \gamma\|u\|^{2}+\frac{8 C^{\prime 2}}{\alpha \beta}|p|^{2}\right\} d s .
\end{gathered}
$$


Considering that

$$
\begin{gathered}
2\left(g_{1}(u), A u\right) \geq-\frac{1}{4}|A u|^{2}-4 \int_{\Omega}\left|g_{1}(u)\right|^{2} d x \\
2(f, A u) \geq-\frac{1}{4}|A u|^{2}-4|f|^{2}, \\
\int_{\Omega}\left|g_{1}(u)\right|^{2} d x \leq \int_{\Omega}\left(1+|u|^{(\rho+1)}\right)^{2} d x \leq 2|\Omega|^{2}+2\|u\|^{2},
\end{gathered}
$$

by the assumption $\left(H_{2}\right)$ of $g_{1}(\cdot)$, we have

$$
\begin{gathered}
|A u|^{2}+\|v\|^{2}+\|\widetilde{\theta}\|^{2} \\
\leq 2 e^{-\alpha \tau}\left(\beta\left|A u_{0}\right|^{2}+\left\|v_{0}\right\|^{2}+\left\|\widetilde{\theta}_{0}\right\|^{2}\right. \\
\left.+2\left(g_{1}\left(u_{0}\right), A u_{0}\right)-2\left(f(t-\tau), A u_{0}\right)\right) \\
+4 \int_{t-\tau}^{t} e^{-\alpha(t-s)}\left\{\frac{4}{\alpha \beta}\left|f^{\prime}\right|^{2}+\frac{2}{k}|Q|^{2}+\frac{2 \alpha_{1}^{2}}{k}|\widetilde{\theta}|^{2}\right. \\
+\frac{4\left(\alpha^{3}+\alpha \gamma^{2}\right)}{\beta}\left(2|p|^{2}+\frac{2 a^{2}}{\lambda}\|u\|^{2}\right) \\
+\left[\left(\frac{\alpha^{2}}{2}+\frac{4 C^{\prime 2}}{\alpha \beta}\right)^{2}\right] \gamma\|u\|^{2} \\
\left.+\frac{8 C^{\prime 2}}{\alpha \beta}|p|^{2}\right\} d s \\
+16|\Omega|^{2}+16\|u\|^{2}+8|f|^{2} .
\end{gathered}
$$

Set

$$
\begin{aligned}
& C_{3}=\max \left\{\frac{8\left(\alpha^{2}+\gamma^{2}\right) \alpha^{3}}{\beta \lambda}+\frac{\alpha^{2} \gamma}{2}+\left(\frac{4 C^{\prime 2}}{\alpha \beta}\right)^{2} \gamma,\right. \\
& \left.\frac{8 \alpha^{4}+8 \alpha^{2} \gamma^{2}+8 C^{\prime 2}}{\alpha \beta}, \frac{2 a_{1}^{2}}{k}\right\}, \\
& C_{4}=\max \left\{\frac{4}{\alpha}, \frac{2}{k}\right\}, \\
& C_{5}=2 \beta+\frac{4 \alpha^{2}+2}{\lambda}+4, \\
& C_{6}=\max \left\{1+\frac{2 \alpha^{2}}{\lambda}, 2\right\} .
\end{aligned}
$$

Since $\delta<\alpha$, we have from (32)

$$
\begin{gathered}
C_{3} \int_{t-\tau}^{t} e^{-\alpha(t-s)}\left\{\|u(s)\|^{2}+|p(s)|^{2}+|\widetilde{\theta}(s)|^{2}\right\} \\
\leq \frac{C_{1} C_{2} C_{3}}{\alpha \lambda} e^{-\delta \tau}\left(\left|A u_{0}\right|^{2}+\left\|p_{0}\right\|^{2}+\left\|\widetilde{\theta}_{0}\right\|^{2}\right) \\
\quad+\frac{2 C_{1} C_{2} C_{3}}{\alpha} e^{-\delta \tau} \phi\left(u_{0}\right)
\end{gathered}
$$

$$
\begin{aligned}
& +\frac{C_{1} C_{3}}{\alpha-\delta} \int_{-\infty}^{t} e^{-\delta(t-s)}\left(\frac{2}{\gamma}|f|^{2}+\frac{2}{\lambda k}|Q|^{2}\right) d s \\
& +\frac{2 C_{1} C_{2} \alpha M}{\alpha \delta},
\end{aligned}
$$

and then we have from (42)

$$
\begin{aligned}
& |A u|^{2}+\|p\|^{2}+\|\widetilde{\theta}\|^{2} \\
& \leq e^{-\alpha \tau}\left(\left(2 \beta+\frac{4 \alpha^{2}+2}{\lambda}+2\right) C_{6}\left|A u_{0}\right|^{2}\right. \\
& \left.+2\left\|p_{0}\right\|^{2}+\left\|\tilde{\theta}_{0}\right\|^{2}\right) \\
& +2 C_{6} e^{-\alpha \tau}\left(|\Omega|^{2}+|f(t-\tau)|^{2}\right) \\
& +\frac{C_{1} C_{2} C_{3} C_{6}}{\alpha \lambda} e^{-\delta \tau}\left(\left|A u_{0}\right|^{2}+\left\|p_{0}\right\|^{2}+\left\|\widetilde{\theta}_{0}\right\|^{2}\right) \\
& +\frac{2 C_{1} C_{2} C_{3} C_{6}}{\alpha} e^{-\delta \tau} \phi\left(u_{0}\right) \\
& +\frac{C_{1} C_{3} C_{6}}{\alpha-\delta} \int_{-\infty}^{t} e^{-\delta}(t-s)\left(\frac{2}{\gamma}|f|^{2}+\frac{2}{\lambda k}|Q|^{2}\right) d s \\
& +\frac{2 M C_{1} C_{3} C_{6}}{\delta}+C_{4} C_{6} \\
& \times \int_{-\infty}^{t} e^{-\alpha(t-s)}\left(|f|^{2}+|Q|^{2}\right) d s+16|\Omega|^{2} \\
& +16\|u\|^{2}+8|f|^{2} \\
& \leq\left(\frac{C_{1} C_{2} C_{3} C_{6}}{\alpha \lambda} e^{-\delta \tau}+C_{5} C_{6} e^{-\alpha \tau}\right) \\
& \times\left(\left|A u_{0}\right|^{2}+\left\|p_{0}\right\|^{2}+\left\|\widetilde{\theta}_{0}\right\|^{2}\right) \\
& +\frac{2 C_{1} C_{2} C_{3} C_{6}}{\alpha} e^{-\delta \tau} \phi\left(u_{0}\right) \\
& +2 C_{6} e^{-\alpha \tau}\left(|\Omega|^{2}+|f(t-\tau)|^{2}\right)+\frac{C_{1} C_{3} C_{6}}{\alpha-\delta} \\
& \times \int_{-\infty}^{t}\left(\frac{2}{\gamma}|f|^{2}+\frac{2}{\lambda k}|Q|^{2}\right) d s+\frac{2 M C_{1} C_{3} C_{6}}{\delta}+C_{4} C_{6} \\
& \times \int_{-\infty}^{t} e^{-\alpha(t-s)}\left(|f|^{2}+|Q|^{2}\right) d s+16 C_{6}|\Omega|^{2} \\
& +16 C_{6}\|u\|^{2}+8 C_{6}|f|^{2} \text {. }
\end{aligned}
$$

Let $\widehat{D} \in D_{\delta, E_{1}}$ be given. For all $y(t-\tau)=y_{0} \in D(t-\tau), t \in R$ and $\tau \geq 0$, from the assumption $\left(H_{1}\right)$ of $g_{1}(\cdot)$, we know that $\phi(u(t-\tau))$ is bounded and positive. So we easily obtain from (45)

$$
\begin{aligned}
& \left\|\Phi\left(t, t-\tau, y_{0}\right)\right\|_{E_{1}}^{2} \\
& \quad \leq\left(\frac{C_{1} C_{2} C_{3} C_{6}}{\alpha \lambda} e^{-\delta \tau}+C_{5} C_{6} e^{-\alpha \tau}\right)
\end{aligned}
$$




$$
\begin{aligned}
& \times\left(\left|A u_{0}\right|^{2}+\left\|p_{0}\right\|^{2}+\left\|\widetilde{\theta}_{0}\right\|^{2}\right)+\frac{2 C_{1} C_{2} C_{3} C_{6}}{\alpha} e^{-\delta \tau} \phi\left(u_{0}\right) \\
& +2 C_{6} e^{-\alpha \tau}\left(|\Omega|^{2}+|f(t-\tau)|^{2}\right) \\
& +\frac{C_{1} C_{3} C_{6}}{\alpha-\delta} \int_{-\infty}^{t}\left(\frac{2}{\gamma}|f|^{2}+\frac{2}{\lambda k}|Q|^{2}\right) d s+\frac{2 M C_{1} C_{3} C_{6}}{\delta} \\
& +C_{4} C_{6} \int_{-\infty}^{t} e^{-\alpha(t-s)}\left(|f|^{2}+|Q|^{2}\right) d s+16 C_{6}|\Omega|^{2} \\
& +16 C_{6}\|u\|^{2}+8 C_{6}|f|^{2}
\end{aligned}
$$

for all $y_{0} \in D(t-\tau), t \in R$, and $\tau \geq 0$. Set

$$
\begin{aligned}
&\left(R_{\delta, E_{1}}(t)\right)^{2} \\
&=2\left\{\frac{C_{1} C_{3} C_{6}}{\alpha-\delta} \int_{-\infty}^{t} e^{-\delta(t-s)}\left(\frac{2}{\gamma}|f|^{2}+\frac{2}{\lambda k}|Q|^{2}\right) d s\right. \\
& \quad+\frac{2 M C_{1} C_{3} C_{6}}{\delta}+C_{4} C_{6} \int_{-\infty}^{t} e^{-\alpha(t-s)}\left(|f|^{2}+|Q|^{2}\right) d s \\
&\left.\quad+16 C_{6}|\Omega|^{2}+16 C_{6}\left(R_{\delta, E_{0}}(t)\right)^{2}+8 C_{6}|f|^{2}\right\} .
\end{aligned}
$$

The family $\widehat{B}_{\delta, E_{1}}$ of closed balls in $E_{1}$

$$
B_{\delta, E_{1}}(t)=\left\{y \in E_{1},\|y\|_{E_{1}} \leq R_{\delta, E_{1}}(t)\right\}
$$

is pullback $D_{\delta, E_{1}}$-absorbing for the cocycle $\Phi$ in $E_{1}$.

\section{The Pullback $D_{\delta, E_{1}}$-Attractor in $E_{1}$}

In order to get the existence of the pullback $D_{\delta, E_{1}}$-attractor, we first introduce the following Lemma.

Lemma 4. Let $H$ be an infinite dimensional Hilbert space and let the family $\left\{\omega_{i}\right\}_{i \in N}$ be an orthonormal basis of $H$. Suppose that $f(x, t), f^{\prime}(x, t), Q(x, t) \in L_{l o c}^{2}(R, H)$ and for any $t \in R$,

$$
\int_{-\infty}^{t} e^{\sigma s}\left(|f(x, s)|_{H}^{2}+\left|f^{\prime}(x, t)\right|_{H}^{2}+|Q(x, s)|_{H}^{2}\right) d s<\infty,
$$$$
\text { for any } \sigma \geq 0 \text {. }
$$

Then

$$
\begin{gathered}
\lim _{n \rightarrow \infty} \int_{-\infty}^{t} e^{\sigma s}\left(\left|\left(I-P_{n}\right) f(x, s)\right|_{H}^{2}+\left|\left(I-P_{n}\right) f^{\prime}(x, s)\right|_{H}^{2}\right. \\
\left.+\left|\left(I-P_{n}\right) Q(x, s)\right|_{H}^{2}\right) d s=0, \\
\forall t \in R,
\end{gathered}
$$

where $P_{n}: H \rightarrow \operatorname{span}\left\{\omega_{1}, \omega_{2}, \ldots, \omega_{n}\right\}$ is the orthogonal projector.
Proof. Let $\eta_{i}(t)=\left(f(x, t), \omega_{i}\right)_{H}, \xi_{i}=\left(f^{\prime}(x, t), \omega_{i}\right)_{H}$, and $\zeta_{i}(t)=\left(Q(x, t), \omega_{i}\right)$, so

$$
\begin{gathered}
f(x, t)=\sum_{i=1}^{\infty} \eta_{i}(t) \omega_{i}, \quad f^{\prime}(x, t)=\sum_{i=1}^{\infty} \xi_{i}(t) \omega_{i}, \\
Q(x, t)=\sum_{i=1}^{\infty} \zeta_{i}(t) \omega_{i} .
\end{gathered}
$$

For any $t \in R$ and any $\varepsilon>0$,

$$
\begin{aligned}
& \int_{-\infty}^{t} e^{\sigma s}\left(|f(x, s)|_{H}^{2}+\left|f^{\prime}(x, s)\right|_{H}^{2}+|Q(x, s)|_{H}^{2}\right) d s \\
& =\sum_{i=1}^{\infty} \int_{-\infty}^{t} e^{\sigma s}\left(\left|\eta_{i}(s)\right|^{2}+\left|\xi_{i}(s)\right|^{2}+\left|\zeta_{i}(t)\right|^{2}\right) d s<\infty
\end{aligned}
$$

we can choose $N_{1}, N_{2}, N_{3}$ large enough so that

$$
\begin{aligned}
& \sum_{i=N_{i}}^{\infty} \int_{-\infty}^{t} e^{\sigma s}\left|\eta_{i}(s)\right|^{2} d s \leq \frac{\varepsilon}{3}, \\
& \sum_{i=N_{i}}^{\infty} \int_{-\infty}^{t} e^{\sigma s}\left|\xi_{i}(s)\right|^{2} d s \leq \frac{\varepsilon}{3}, \\
& \sum_{i=N_{i}}^{\infty} \int_{-\infty}^{t} e^{\sigma s}\left|\zeta_{i}(s)\right|^{2} d s \leq \frac{\varepsilon}{3} .
\end{aligned}
$$

Then for any $t \in R$ and any $\varepsilon>0$, we put $N_{0}=$ $\max \left\{N_{1}, N_{2}, N_{3}\right\}$ to get

$$
\sum_{i=N_{0}}^{\infty} \int_{-\infty}^{t} e^{\sigma s}\left(\left|\eta_{i}(s)\right|^{2}+\left|\xi_{i}(s)\right|^{2}+\left|\zeta_{i}(s)\right|^{2}\right) d s \leq \varepsilon
$$

That is, for any $t \in R$, any $\varepsilon>0$, and $n \geq N_{0}$,

$$
\begin{gathered}
\int_{-\infty}^{t} e^{\sigma s}\left(\left|\left(I-P_{n}\right) f(x, s)\right|_{H}^{2}+\left|\left(I-P_{n}\right) f^{\prime}(x, s)\right|_{H}^{2}\right. \\
\left.+\left|\left(I-P_{n}\right) Q(x, s)\right|_{H}^{2}\right) d s \leq \varepsilon .
\end{gathered}
$$

So

$$
\begin{gathered}
\lim _{n \rightarrow \infty} \int_{-\infty}^{t} e^{\sigma s}\left(\left|\left(I-P_{n}\right) f(x, s)\right|_{H}^{2}+\left|\left(I-P_{n}\right) f^{\prime}(x, s)\right|_{H}^{2}\right. \\
\left.+\left|\left(I-P_{n}\right) Q(x, s)\right|_{H}^{2}\right) d s=0 .
\end{gathered}
$$

In order to obtain the pullback $D_{\delta, E_{1}}$-attractor in $E_{1}$, we also need the following Lemmas of the properties of compactness about the nonlinear operator $g_{1}(\cdot), g_{2}(\cdot)$.

Lemma 5. Let $g_{1}(\cdot)$ be a $C^{2}(R, R)$ function from $R$ into $R$ satisfying $\left(H_{2}\right)$; then $g_{1}: D(A) \rightarrow H_{0}^{1}(\Omega)$ is continuously compact; that is, $g_{1}(\cdot)$ is continuous and maps a bounded subset of $D(A)$ into a precompact subset of $H_{0}^{1}(\Omega)$. 
Proof. Let $B=B_{D(A)}$ be a bounded set in $D(A)$. Assume that $\left\{u_{n}\right\}$ is a bounded sequence in $B$. From Sobolev embedding Theorem, the embeddings $D(A) \mapsto L^{p}, \forall p \geq 1$ and $D(A) \mapsto w^{1, p}(\forall p \geq 1)$ are compact. We assume that $\left\{u_{n}\right\}$ is bounded and converges to $u_{0}$ in $L^{p}$ and $W^{1, p}$, respectively. By Minkowski inequality, we see that

$$
\begin{aligned}
& \left(\int_{\Omega}\left|\nabla\left(g_{1}\left(u_{n}\right)-g_{1}\left(u_{0}\right)\right)\right|^{2} d x\right)^{1 / 2} \\
& \leq\left\{\int_{\Omega}\left[g_{1}^{\prime}\left(u_{n}\right)-g_{1}^{\prime}\left(u_{0}\right) \nabla u_{n}\right]^{2} d x\right\}^{1 / 2} \\
& +\left\{\int_{\Omega}\left[g_{1}^{\prime}\left(u_{0}\right) \nabla\left(u_{n}-u_{0}\right)\right]^{2} d x\right\}^{1 / 2} .
\end{aligned}
$$

By Holder inequality, we have

$$
\begin{aligned}
& \left\{\int_{\Omega}\left[g_{1}^{\prime}\left(u_{n}\right)-g_{1}^{\prime}\left(u_{0}\right)\right]^{2} d x\right\}^{1 / 2} \\
& \quad \leq\left(\int_{\Omega}\left|g_{1}^{\prime}\left(u_{n}\right)-g_{1}^{\prime}\left(u_{0}\right)\right|^{2 p} d x\right)^{1 / 2 p}\left(\int_{\Omega}\left|\nabla u_{n}\right|^{2 q} d x\right)^{1 / 2 q} \\
& \quad \leq C\left(R_{\delta, E_{1}}(t)\right)^{2}\left|g_{1}^{\prime}\left(u_{n}\right)-g_{1}^{\prime}\left(u_{0}\right)\right|_{L^{2 p}}
\end{aligned}
$$

where $1 / p^{\prime}+1 / q^{\prime}+1 / 2=1$ and $C\left(R_{\delta, E_{1}}^{2}(t)\right)$ is a constant depending on $R_{\delta, E_{1}}^{2}(t)$ and the embedding constant. Due to the assumption $\left(H_{3}\right)$ of $g_{1}(\cdot)$ and a classical continuity result, it follows that

$$
\left|g_{1}^{\prime}\left(u_{n}\right)-g_{1}^{\prime}\left(u_{0}\right)\right|_{L^{2 p}} \longrightarrow 0 .
$$

Also by the Holder inequality

$$
\left\{\int_{\Omega}\left[g_{1}^{\prime}\left(u_{0}\right) \nabla\left(u_{n}-u_{0}\right)\right]^{2} d x\right\}^{1 / 2} \longrightarrow 0 .
$$

The proof is completed.

Lemma 6. Let $g_{2}(\cdot)$ be a $C^{1}(R, R)$ function from $R$ into $R$ satisfying (11); then $g_{2}: H_{0}^{1}(\Omega) \rightarrow L^{2}(\Omega)$ is continuously compact.

Proof. Let $B=B_{H_{0}^{1}(\Omega)}$ be a bounded set in $H_{0}^{1}(\Omega)$ and assume $\{\widetilde{\theta}\}$ to be a bounded sequence in $B$. From Sobolev embedding theorem, the embedding $H_{0}^{1}(\Omega) \mapsto L^{p}, \forall p \geq 1$ is compact, so we assume that $\widetilde{\theta}_{n}$ is bounded and converges to $\widetilde{\theta}_{0}$ in $L^{p}$. Let $\tilde{\theta}_{n}-\tilde{\theta}_{0}=\omega_{n}$; then there exists $\theta=\theta(x) \in[0,1]$ such that

$$
\begin{aligned}
& \left(\int_{\Omega}\left|\left(g_{2}\left(\widetilde{\theta}_{n}\right)-g_{2}\left(\widetilde{\theta}_{0}\right)\right)\right|^{2} d x\right)^{1 / 2} \\
& \quad=\left\{\int_{\Omega}\left[g_{2}^{\prime}\left(\widetilde{\theta}_{0}+\theta \omega_{n}\right) \omega_{n}\right]^{2} d x\right\}^{1 / 2} .
\end{aligned}
$$

By Holder inequality, we have

$$
\begin{gathered}
\left(\int_{\Omega}\left|\left(g_{2}\left(\widetilde{\theta}_{n}\right)-g_{2}\left(\widetilde{\theta}_{0}\right)\right)\right|^{2} d x\right)^{1 / 2} \\
\quad \leq\left|g_{2}^{\prime}\left(\widetilde{\theta}_{0}+\theta \omega_{n}\right)\right|_{L^{2 p^{\prime}}}\left|\omega_{n}\right|_{L^{2 q^{\prime}}}
\end{gathered}
$$

where $q^{\prime}$ is the conjugate of $p^{\prime}$ (i.e., $1 / p^{\prime}+1 / q^{\prime}=1$ ). Combined with the assumption (11), the proof is completed.

Lemma 7. Let $g_{1}(\cdot)$ be a $C^{2}(R, R)$ function from $R$ into $R$ satisfying $\left(H_{2}\right)$; moreover, $g_{1}(0)=0$. Let $B$ be a bounded subset of $D(A)$. Then for any $\varepsilon>0$, there exists some $n_{0}$ such that when $n \geq n_{0}$

$$
\left\|\left(I-p_{n}\right) g_{1}(u)\right\| \leq \varepsilon, \quad \forall u \in B,
$$

where $P_{n}: V \rightarrow \operatorname{span}\left\{\omega_{1}, \omega_{2}, \ldots, \omega_{n}\right\}$ is the orthogonal projection.

Proof. Note that $g_{1}(u) \in L^{2}(\Omega)$ for $u \in D(A)$. By Lemma 5, we see that $g_{1}(\cdot)$ maps bounded subsets of $D(A)$ into precompact subsets of $H_{0}^{1}(\Omega)$. Let $B$ be a bounded subset of $D(A)$ and let $\varepsilon>0$ be given arbitrarily. Since $g_{1}(B)$ is precompact in $H_{0}^{1}(\Omega)$, there is a finite number of elements $v_{1}, v_{2}, \ldots, v_{k} \in g_{1}(B)$ such that

$$
g_{1}(B) \subset \bigcup_{1 \leq i \leq k} B\left(v_{i}, \frac{\varepsilon}{2}\right) .
$$

We take $n_{0}>0$ sufficiently large so that

$$
\left\|\left(I-P_{n}\right) g_{1}(u)\right\| \leq \varepsilon, \quad \forall u \in B
$$

for all $1 \leq i \leq k$, when $n \geq n_{0}$. Then by $g_{1}(B) \subset_{1 \leq i \leq k} B\left(v_{i}, \varepsilon / 2\right)$, we have

$$
\left\|\left(I-P_{n}\right) g_{1}(u)\right\| \leq \varepsilon, \quad \forall u \in V,
$$

where $P_{n}: D(A) \rightarrow \operatorname{span}\left\{\omega_{1}, \ldots, \omega_{n}\right\}$ is the orthogonal projection.

Lemma 8. Let $g_{2}(\cdot)$ be a $C^{2}(R, R)$ function from $R$ into $R$ satisfying (11). Let $B$ be a bounded subset of $H_{0}^{1}(\Omega)$. Then for any $\varepsilon>0$, there exists some $n_{0}$ such that when $n \geq n_{0}$

$$
\left\|\left(I-p_{n}\right) g_{2}(\widetilde{\theta})\right\| \leq \varepsilon, \quad \forall \widetilde{\theta} \in B,
$$

where $P_{n}: H_{0}^{1}(\Omega) \rightarrow \operatorname{span}\left\{\omega_{1}, \omega_{2}, \ldots, \omega_{n}\right\}$ is the orthogonal projection.

Theorem 9. Assume that $\beta, \gamma, k>0$ and the assumptions of $g_{1}(\cdot), g_{2}(\cdot), h(\cdot)$ hold. $f(x, t) \in L_{l o c}^{2}\left(R, L^{2}(\Omega)\right), f^{\prime}(x, t) \in$ $L_{\text {loc }}^{2}\left(R, L^{2}(\Omega)\right)$, and $Q(x, t) \in L_{\text {loc }}^{2}\left(R, L^{2}(\Omega)\right)$ satisfy (7); then there exists a pullback $D_{\delta, E_{1}}$-attractor in $E_{1}$ for the nonautonomous dynamical system $(\theta, \Phi)$ defined by $(15)$.

Proof. In order to prove the result of the theorem, we only need to check the pullback $D_{\delta, E_{1}}$-condition (C).

Let $\left\{\omega_{k}\right\}_{k=1}^{\infty}$ be an orthonormal basis of $H$ which consists of eigenvectors of $A$. The corresponding eigenvalues are denoted by $\lambda_{k}, k=1,2, \ldots$ and $0<\lambda_{1} \leq \lambda_{2} \leq \lambda_{3} \leq \ldots$, $\lambda_{j} \rightarrow \infty$, as $j \rightarrow \infty$. Then $\left\{\omega_{k}\right\}_{k=1}^{\infty}$ is also an orthonormal basis of $V$ and $D(A)$. We write $V_{n}=\left\{\omega_{1}, \omega_{2}, \ldots, \omega_{n}\right\}$ and $P_{n}: V \rightarrow V_{n}$ is an orthogonal projector. For any $u \in V$, we write

$$
u=P_{n} u+\left(I-P_{n}\right) u \triangleq u_{1}+u_{2} .
$$


Take the scalar product in $H$ of (1) with $A v_{2}=A u_{2}^{\prime}+$ $\alpha A u_{2}$, and take the scalar product in $H$ of (2) with $A \widetilde{\theta}_{2}$; then make summation to get

$$
\begin{aligned}
\frac{1}{2} \frac{d}{d r} & \left(\beta\left|A u_{2}\right|^{2}+\left\|v_{2}\right\|^{2}+\left\|\widetilde{\theta}_{2}\right\|^{2}\right)+\gamma\left\|v_{2}\right\|^{2}-\alpha\left\|v_{2}\right\|^{2} \\
& +\alpha \beta\left|A u_{2}\right|^{2}+k\left|A \widetilde{\theta}_{2}\right|^{2}+\alpha^{2}\left(\left(u_{2}, v_{2}\right)\right) \\
& -\gamma \alpha\left(A u_{2}, v_{2}\right)+\alpha\left(A^{1 / 2} \widetilde{\theta}_{2}, A u_{2}\right) \\
& +\left(\left(\left(I-P_{n}\right) g_{1}(u), v_{2}\right)\right) \\
= & \left(\left(I-P_{n}\right) g_{2}(\widetilde{\theta}), A \widetilde{\theta}_{2}\right)+\left(\left(I-P_{n}\right) f, A v_{2}\right) \\
& +\left(\left(I-P_{n}\right) Q, A \widetilde{\theta}_{2}\right) .
\end{aligned}
$$

Since

$$
\begin{aligned}
& \alpha^{2}\left(\left(u_{2}, v_{2}\right)\right) \geq-\frac{\alpha^{2}}{2}\left\|u_{2}\right\|^{2}-\frac{\alpha^{2}}{2}\left\|v_{2}\right\|^{2} ; \\
& -\gamma \alpha\left(A u_{2}, v_{2}\right) \geq-\frac{\alpha \gamma^{2}}{\beta}\left|v_{2}\right|^{2}-\frac{\alpha \beta}{4}\left|A u_{2}\right|^{2} ; \\
& \alpha\left(A^{1 / 2} \widetilde{\theta}_{2}, A u_{2}\right) \geq-\frac{\alpha}{\beta}\left\|\widetilde{\theta}_{2}\right\|^{2}-\frac{\alpha \beta}{4}\left|A u_{2}\right|^{2}, \\
& \left|\left(\left(I-P_{n}\right) g_{1}(u), v_{2}\right)\right| \leq \frac{1}{\gamma}\left\|\left(I-P_{n}\right) g_{1}(u)\right\|^{2}+\frac{\gamma}{4}\left\|v_{2}\right\|^{2} ; \\
& \left|\left(\left(I-P_{n}\right) g_{2}(\widetilde{\theta}), A \widetilde{\theta}_{2}\right)\right| \leq \frac{1}{k}\left\|\left(I-P_{n}\right) g_{2}(\widetilde{\theta})\right\|^{2}+\frac{k}{4}\left\|A \widetilde{\theta}_{2}\right\|^{2} ; \\
& \left|\left(\left(I-P_{n}\right) f, A v_{2}\right)\right| \\
& \quad=\frac{d}{d t}\left(f_{2}, A u_{2}\right)+\alpha\left(f_{2}, A u_{2}\right)-\left(f^{\prime}, A u_{2}\right) \\
& \quad \leq \frac{d}{d t}\left(f_{2}, A u_{2}\right)+\frac{\alpha \beta}{4}\left|A u_{2}\right|^{2}+\frac{2 \alpha}{\beta}\left|f_{2}\right|^{2}+\frac{2}{\alpha \beta}\left|f_{2}^{\prime}\right|^{2}
\end{aligned}
$$

here we set $f_{2}=\left(I-P_{n}\right) f$, and

$$
\left|\left(\left(I-P_{n}\right) Q, A \widetilde{\theta}_{2}\right)\right| \leq \frac{1}{k}\left|\left(I-P_{n}\right) Q\right|^{2}+\frac{k}{4}\left|A \widetilde{\theta}_{2}\right|^{2},
$$

and letting $0 \leq \alpha \leq \min \left\{\beta \lambda / 4,\left(\left(-\lambda+\gamma^{2} / \beta\right)+\right.\right.$ $\left.\sqrt{\left.\left(\lambda+\gamma^{2} / \beta\right)^{2}+\gamma \lambda^{2} / 2\right)} / \lambda, k \lambda \beta / 2\right\}$, setting $\alpha_{1}=\min \{\alpha / 8$, $\gamma \lambda / 2, k \lambda / 4\}$, we have, from (69),

$$
\begin{aligned}
& \frac{d}{d r}\left(\beta\left|A u_{2}-\frac{1}{\beta} f_{2}\right|^{2}+\left\|v_{2}\right\|^{2}+\left\|\widetilde{\theta}_{2}\right\|^{2}\right) \\
& \quad+2 \alpha_{1}\left(\beta\left|A u_{2}-\frac{1}{\beta} f_{2}\right|^{2}+\left\|v_{2}\right\|^{2}+\left\|\widetilde{\theta}_{2}\right\|^{2}\right)
\end{aligned}
$$

$$
\begin{aligned}
\leq & \frac{2}{\gamma}\left\|\left(I-P_{n}\right) g_{1}(u)\right\|^{2}+\frac{2}{k}\left|\left(I-P_{n}\right) g_{1}(\tilde{\theta})\right|^{2} \\
& +\frac{2}{k}\left|\left(I-P_{n}\right) Q\right|^{2}+\frac{2 \alpha}{\beta}\left|\left(I-P_{n}\right) f_{2}\right|^{2} \\
& +\frac{2}{\alpha \beta}\left|\left(I-P_{n}\right) f_{2}^{\prime}\right|^{2}+\frac{2}{\beta}\left(f_{2}, f_{2}^{\prime}\right)+\frac{2 \alpha_{1}}{\beta}\left|f_{2}\right|^{2} .
\end{aligned}
$$

By the Gronwall lemma, we obtain

$$
\begin{gathered}
\beta\left|A u_{2}-\frac{1}{\beta} f_{2}\right|^{2}+\left\|v_{2}\right\|^{2}+\left\|\tilde{\theta}_{2}\right\|^{2} \\
\leq e^{-2 \alpha_{1} \tau}\left[\beta\left|A u_{2}(t-\tau)-\frac{1}{\beta} f_{2}(t-\tau)\right|^{2}\right. \\
\left.+\left\|v_{2}(t-\tau)\right\|^{2}+\left\|\widetilde{\theta}_{2}(t-\tau)\right\|^{2}\right] \\
+\int_{t-\tau}^{t} e^{-2 \alpha_{1}(t-s)}\left(\frac{2}{\gamma}\left\|\left(I-P_{n}\right) g_{1}(u)\right\|^{2}\right. \\
+\frac{2}{k}\left|\left(I-P_{n}\right) g_{1}(\widetilde{\theta})\right|^{2}+\frac{2}{k}\left|\left(I-P_{n}\right) Q\right|^{2} \\
+\frac{2 \alpha}{\beta}\left|\left(I-P_{n}\right) f_{2}\right|^{2}+\frac{2}{\alpha \beta}\left|\left(I-P_{n}\right) f_{2}^{\prime}\right|^{2} \\
\left.+\frac{2}{\beta}\left(f_{2}, f_{2}^{\prime}\right)+\frac{2 \alpha_{1}}{\beta}\left|f_{2}\right|^{2}\right) d s .
\end{gathered}
$$

So

$$
\begin{gathered}
\left|A u_{2}\right|^{2}+\left\|p_{2}\right\|^{2}+\left\|\widetilde{\theta}_{2}\right\|^{2} \\
\leq 2 e^{-2 \alpha_{1} \tau}\left[2\left(\beta^{2}+\frac{2 \alpha^{2}}{\lambda}\right)\left|A u_{2}(t-\tau)\right|^{2}+2\left\|p_{2}(t-\tau)\right\|^{2}\right. \\
\left.+\left\|\widetilde{\theta}_{2}(t-\tau)\right\|^{2}+\frac{2}{\beta}\left|f_{2}(t-\tau)\right|^{2}\right] \\
+2 \int_{t-\tau}^{t} e^{-2 \alpha_{1}(t-s)}\left(\frac{2}{\gamma}\left\|\left(I-P_{n}\right) g_{1}(u)\right\|^{2}\right. \\
+\frac{2}{k}\left|\left(I-P_{n}\right) g_{1}(\widetilde{\theta})\right|^{2} \\
+\frac{2}{k}\left|\left(I-P_{n}\right) Q\right|^{2} \\
+\frac{2 \alpha}{\beta}\left|\left(I-P_{n}\right) f_{2}\right|^{2} \\
+\frac{2}{\alpha \beta}\left|\left(I-P_{n}\right) f_{2}^{\prime}\right|^{2}
\end{gathered}
$$




$$
\begin{aligned}
+ & \left.\frac{2}{\beta}\left(f_{2}, f_{2}^{\prime}\right)+\frac{2 \alpha_{1}}{\beta}\left|f_{2}\right|^{2}\right) d s \\
+2 \alpha^{2}\left\|u_{2}\right\|^{2}+ & \frac{2}{\beta}\left|f_{2}\right|^{2} .
\end{aligned}
$$

Then given any $\widehat{D} \in D_{\delta, E_{1}}$, we have

$$
\begin{aligned}
& \left\|\Phi_{2}\left(\tau, t-\tau, y_{0}\right)\right\|_{E_{1}}^{2} \\
& \leq 2 e^{-2 \alpha_{1} \tau}\left[2\left(\beta^{2}+\frac{2 \alpha^{2}}{\lambda}\right)\left|A u_{2}(t-\tau)\right|^{2}+2\left\|p_{2}(t-\tau)\right\|^{2}\right. \\
& \left.+\left\|\widetilde{\theta}_{2}(t-\tau)\right\|^{2}+\frac{2}{\beta}\left|f_{2}(t-\tau)\right|^{2}\right] \\
& +2 \int_{t-\tau}^{t} e^{-2 \alpha_{1}(t-s)}\left(\frac{2}{\gamma}\left\|\left(I-P_{n}\right) g_{1}(u)\right\|^{2}\right. \\
& \left.+\frac{2}{k}\left|\left(I-P_{n}\right) g_{1}(\widetilde{\theta})\right|^{2}\right) d s \\
& +2 \int_{-\infty}^{t}\left(\frac{2}{k}\left|\left(I-P_{n}\right) Q\right|^{2}+\frac{2 \alpha+4+2 \alpha_{1}}{\beta}\left|\left(I-P_{n}\right) f_{2}\right|^{2}\right. \\
& \left.+\left(\frac{2}{\alpha \beta}+\frac{4}{\beta}\right)\left|\left(I-P_{n}\right) f_{2}^{\prime}\right|^{2}\right) d s \\
& +2 \alpha^{2}\left\|u_{2}\right\|^{2}+\frac{2}{\beta}\left|f_{2}\right|^{2} \\
& :=I_{1}+I_{2}+I_{3}+I_{4}
\end{aligned}
$$

for any $y(t-\tau)=y_{0} \in D(t-\tau)$ and $t \in R, \tau \geq 0$. Now we estimate $I_{1}, I_{2}, I_{3}, I_{4}$ one by one. Given any $\varepsilon>0$ and any $t \in R$, it is easy to see that

$$
\frac{2}{\beta}\left|f_{2}\right|^{2} \longrightarrow 0
$$

so there exists $\tau_{1} \geq 0$ such that

$$
I_{1} \leq \frac{\varepsilon}{4}
$$

for all $\tau \geq \tau_{1}, y_{0} \in D(t-\tau)$.

By Lemmas 7-8, we can choose $n_{1} \in N$ such that

$$
I_{2} \leq \frac{\varepsilon}{4}
$$

for any $n \geq n_{1}, \tau \geq \tau_{2}$.

By Lemma 4 , we can choose $n_{2}$ large enough such that

$$
I_{3} \leq \frac{\varepsilon}{4}
$$

for $n \geq n_{2}$.

By (32), there exists $\tau_{3}>0$ such that, for $\tau>\tau_{3}$, $\|u(t)\|^{2}<\infty$ and the embedding from $D(A)$ into $V$ is compact combined with $(2 / \beta)\left|f_{2}\right|^{2} \rightarrow 0$, so we can choose $n_{3}$ large enough such that

$$
I_{4} \leq \frac{\varepsilon}{4}
$$

for $n \geq n_{3}, \tau>\tau_{3}$.

By above analysis, if we choose $\tau_{0}=\max \left\{\tau_{1}, \tau_{2}, \tau_{3}\right\}, n_{0}=$ $\max \left\{n_{1}, n_{2}, n_{3}\right\}$, then

$$
\begin{gathered}
\qquad \Phi_{2}\left(\tau, t-\tau, y_{0}\right) \|_{E_{1}}^{2} \leq \varepsilon \\
\text { for any } \tau \geq \tau_{0}, \quad n \geq n_{0}, \quad y_{0} \in D(t-\tau) .
\end{gathered}
$$

This implies pullback $D_{\delta, E_{1}}$-condition (C).

\section{Conflict of Interests}

The authors declare that there is no conflict of interests regarding the publication of this paper.

\section{Acknowledgments}

The project is supported by the National Natural Science Foundation of China (Grant no. 10772131), the Natural Science Foundation of Shanxi Province, China (Grant no. 2010011008), and the Natural Science Foundation for Young Scientists of Shanxi Province, China (Grant no. 20110210022).

\section{References}

[1] V. Chepyzhov M Vishik, "Attractors for equations of mathematical physics," in Proceedings of the American Mathematical Society Colloquium, vol. 49, AMS, Providence, RI, USA, 2002.

[2] T. Caraballo, G. Łukaszewicz, and J. Real, "Pullback attractors for asymptotically compact non-autonomous dynamical systems," Nonlinear Analysis: Theory, Methods \& Applications, vol. 64, no. 3, pp. 484-498, 2006.

[3] Y. Wang and C. Zhong, "Pullback D-attractors for nonautonomous sine-Gordon equations," Nonlinear Analysis: Theory, Methods \& Applications, vol. 67, no. 7, pp. 2137-2148, 2007.

[4] H. Song, "Pullback attractors of non-autonomous reactiondiffusion equations in $H_{0}^{1}$," Journal of Differential Equations, vol. 249, no. 10, pp. 2357-2376, 2010.

[5] C. T. Anh and T. Q. Bao, "Pullback attractors for generalized Korteweg-de Vries-Burgers equations," Journal of Mathematical Analysis and Applications, vol. 388, no. 2, pp. 899-912, 2012.

[6] S. H. Park and J. Y. Park, "Pullback attractor for a nonautonomous modified Swift-Hohenberg equation," Computers and Mathematics with Applications, vol. 67, pp. 542-548, 2014.

[7] J. Y. Park and J. R. Kang, "Pullback D-attractors for nonautonomous suspension bridge equations," Nonlinear Analysis: Theory, Methods \& Applications, vol. 71, no. 10, pp. 4618-4623, 2009.

[8] Q. Z. Ma and C. K. Zhong, "Existence of global attractors for the suspension bridge equations," Journal of Sichuan University, vol. 43, no. 2, pp. 1-8, 2006.

[9] Q.Z. Ma, C. Y. Sun, and C. K. Zhong, "Existence of strong global attractors for the nonlinear beam equations," Acta Mathematica Scientia, vol. 27, no. 5, pp. 941-948, 2007. 
[10] N. S. Al-Huniti, M. A. Al-Nimr, and M. Naji, "Dynamic response of a rod due to a moving heat source under the hyperbolic heat conduction model," Journal of Sound and Vibration, vol. 242, no. 4, pp. 629-640, 2001.

[11] D. E. Carlson, "Linear thermoelasticity," in Handbuch Physik, vol. 2, pp. 297-346, Springer, Berlin, Germany, 1972.

[12] R. Temam, Infinite-Dimensional Dynamical Systems in Mechanics and Physics, Springer, New York, NY, USA, 1997.

[13] J. M. Ball, "Stability theory for an extensible beam," Journal of Differential Equations, vol. 14, no. 3, pp. 399-418, 1973.

[14] J. M. Ball, "Initial-boundary value problems for an extensible beam," Journal of Mathematical Analysis and Applications, vol. 42, no. 1, pp. 61-90, 1973. 


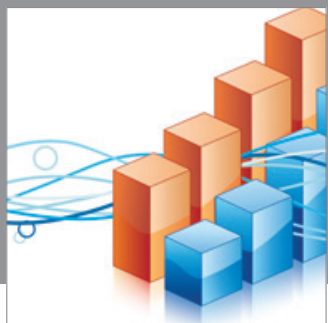

Advances in

Operations Research

mansans

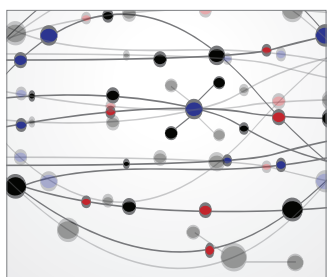

The Scientific World Journal
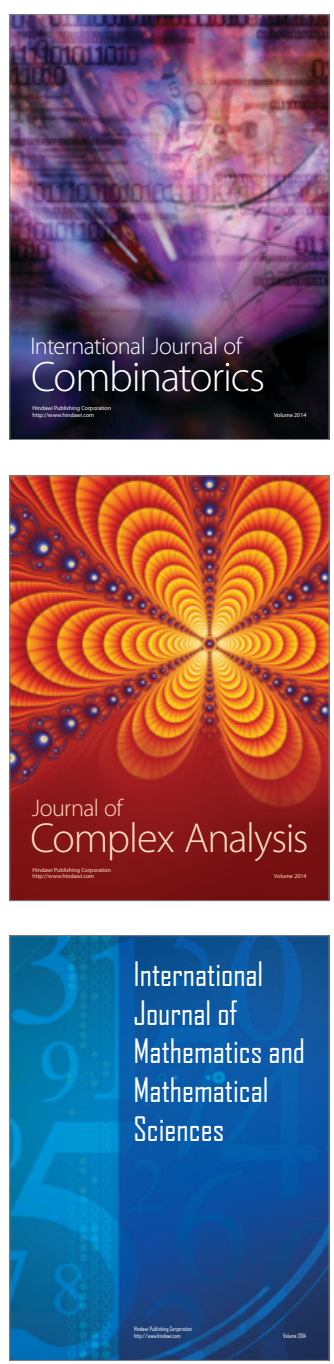
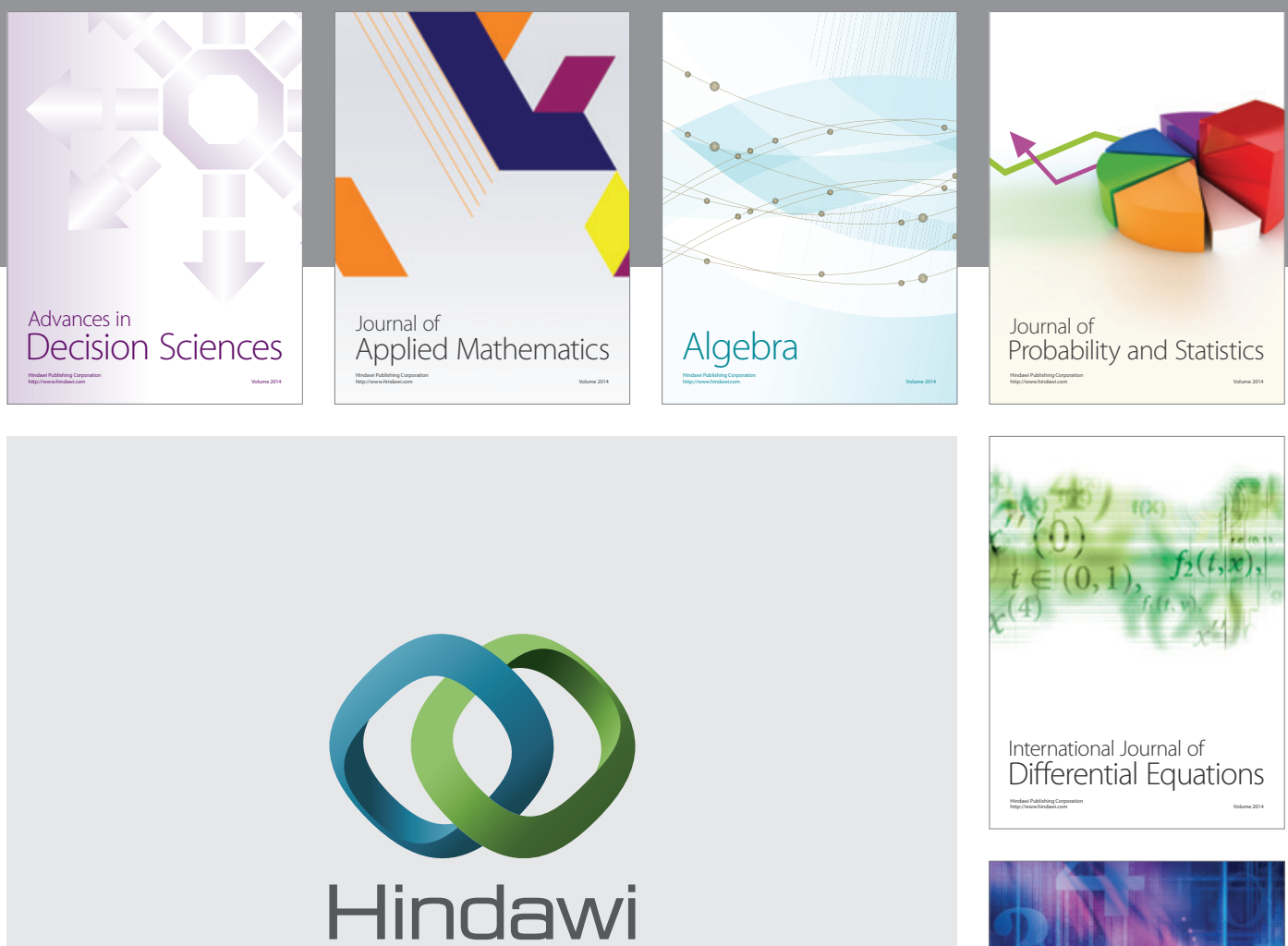

Submit your manuscripts at http://www.hindawi.com
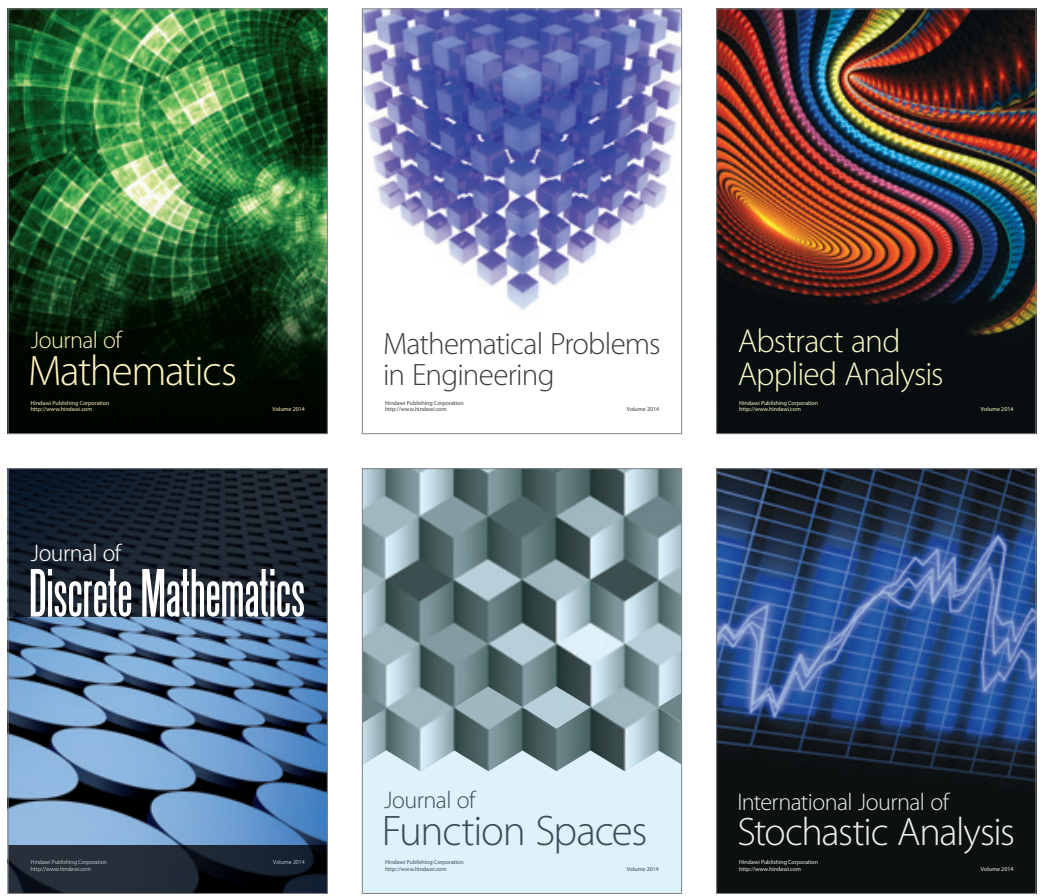

Journal of

Function Spaces

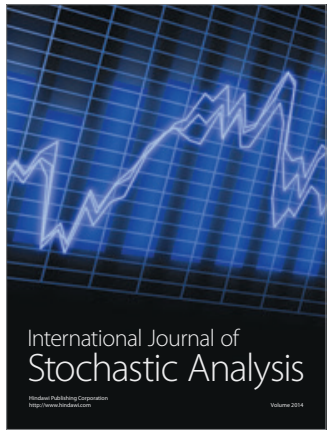

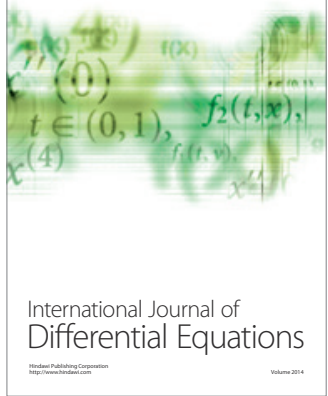
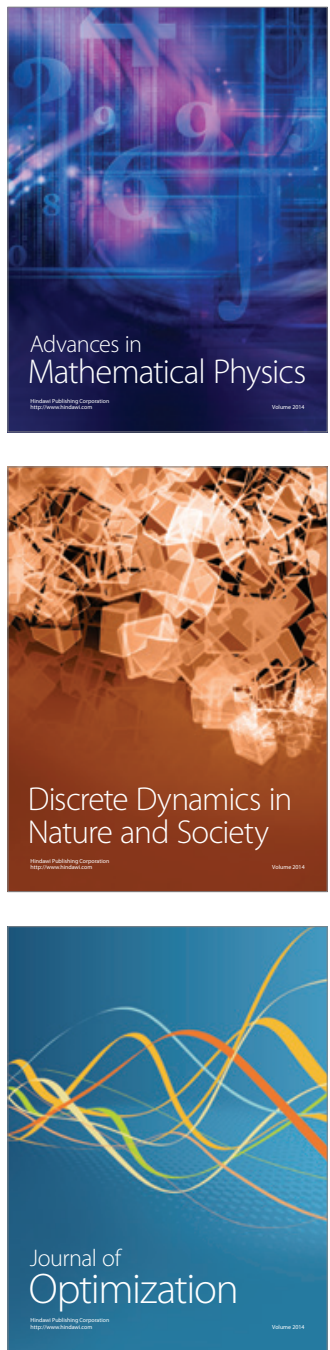